\title{
Efficacy and safety of nivolumab combined with brentuximab vedotin after nivolumab monotherapy failure in patients with relapsed and refractory classic Hodgkin lymphoma
}

\author{
Liudmila V. Fedorova ${ }^{1}$ (1) $\cdot$ Kirill V. Lepik ${ }^{1} \cdot$ Nikita P. Volkov $^{1} \cdot$ Polina V. Kotselyabina ${ }^{1}$. Evgenia S. Borzenkova ${ }^{1}$. \\ Marina O. Popova ${ }^{1} \cdot$ Anastasia V. Beynarovich ${ }^{1} \cdot$ Vadim V. Baykov $^{1} \cdot$ Andrey V. Kozlov $^{1} \cdot$ Ivan S. Moiseev $^{1}$. \\ Natalia B. Mikhailova ${ }^{1} \cdot$ Alexander D. Kulagin ${ }^{1}$
}

Received: 13 August 2021 / Accepted: 10 November 2021 / Published online: 26 November 2021

(c) The Author(s) under exclusive licence to Japan Society of Clinical Oncology 2021

\begin{abstract}
Objectives Therapy of patients with relapsed and refractory classic Hodgkin lymphoma ( $\mathrm{r} / \mathrm{r} \mathrm{cHL})$ after PD-1 inhibitors failure remains an unresolved issue. The aim of this study was to evaluate the efficacy and safety of the combination of nivolumab with brentuximab vedotin (Nivo + BV) after nivolumab monotherapy failure.

Methods This study retrospectively analyzed 21 patients with $\mathrm{r} / \mathrm{r} \mathrm{cHL}$ who were treated with the combination of Nivo + BV after Nivo failure. The response was evaluated by PET-CT scan according to the LYRIC criteria. Adverse events (AEs) were assessed according to NCI CTCAE v.4.03.

Results Median follow-up was 19 (9-47) months. The ORR was 57\%. The median OS was not reached, 24 month OS was 80\% (95\% CI 50-93\%). Median PFS was 12 months with 24 month PFS of 31\% (95\% CI 12-53\%). Any grade AEs were observed in 12 patients (63\%), 3-4 grade AEs in 2 patients (10\%). Allogeneic hematopoietic stem cell transplantation (alloHSCT) after Nivo + BV was performed in $8(38 \%)$ patients. The median time between Nivo + BV and allo-HSCT was 8 (5-21) months.

Conclusions Combination of Nivo $+\mathrm{BV}$ in $\mathrm{r} / \mathrm{r} \mathrm{cHL}$ after nivolumab monotherapy failure is potentially an effective and safe approach.
\end{abstract}

Keywords Hodgkin lymphoma - Relapsed and refractory disease · PD-1 inhibitors · Nivolumab · Brentuximab vedotin · Immunotherapy

\section{Introduction}

Classic Hodgkin lymphoma (cHL) is one of the most common lymphoid cancers with a peak incidence in the age of 20-34 years [1]. The majority of cHL patients have a favorable prognosis; however, up to $30 \%$ patients relapse or have refractory $(\mathrm{r} / \mathrm{r})$ disease to front-line therapy [2-4]. Only 50\% of this group achieves long-term disease control after second-line chemotherapy followed by autologous stem cell transplantation (ASCT) [5-8]. The knowledge about the biology of tumor cells and the microenvironment $[9,10]$ has

Liudmila V. Fedorova

MD.FedorovaL@gmail.com

1 RM Gorbacheva Research Institute, Pavlov University, 6-8 L'va Tolstogo, 197022 St. Petersburg, Russia allowed the creation of new drugs that radically changed the prognosis of patients with cHL [11, 12].

A significant advance in the treatment of $\mathrm{r} / \mathrm{r} \mathrm{cHL}$ was the introduction of brentuximab vedotin (BV), anti-CD30 antibody-drug conjugate that selectively delivers an antimicrotubule agent monomethyl auristatin $\mathrm{E}$ into CD30-expressing cells $[13,14]$. Monotherapy with BV has demonstrated an overall response rate (ORR) of $72 \%$ and complete response (CR) rate of $33 \%$ in patients with $\mathrm{r} / \mathrm{r} \mathrm{cHL}$ [15]. Nevertheless, less than $10 \%$ of patients maintain a long-term remission after BV therapy [16]. The next revolutionary step in the treatment of $\mathrm{r} / \mathrm{r} \mathrm{cHL}$ was the advent of immune checkpoint inhibitors [17]. PD-1 inhibitors nivolumab (Nivo) and pembrolizumab have shown previously unattainable results in the treatment of $\mathrm{r} / \mathrm{r}$ cHL both in clinical trials $[18,19]$ and real clinical practice [20, 21]. 
Despite the success of immunotherapy in patients with $\mathrm{r} / \mathrm{r}$ cHL, more than $60 \%$ of patients have disease relapse or progression during or after PD- 1 inhibitor therapy. Thus, the issue of therapy after immune checkpoint inhibitors failure is unresolved. Allogeneic hematopoietic stem cell transplantation (allo-HSCT) and other cellular therapy methods are possible options for overcoming the resistance in patients with $\mathrm{r} / \mathrm{r}$ cHL $[22,23]$. However, allo-HSCT can only be considered in a limited group of patients due to inadequate response as well as donor unavailability or comorbidity. The optimal approaches of bridge therapy to prepare patients for allo-HSCT are actively being studied. Several options have already demonstrated their efficacy, including the combination of PD-1 inhibitors with chemotherapy [24, 25] and BV with chemotherapy $[26,27]$. The feasibility of conducting these clinical studies was based on preclinical data demonstrating an increase of tumor antigenicity and immunogenicity after pre-treatment with chemotherapy promoting adaptive immune responses, as well as the chemotherapyinduced PD-L1 overexpression in solid tumor patients [28-31]. Considering this data, the possibility of combining PD-1 inhibitors with BV is undoubtedly interesting. Available studies investigated the role of this combination before ASCT as the first salvage therapy [32,33] and after ASCT failure [34-38]. This combination also was studied in patients who had previously received BV monotherapy [34-37, 39]. But only a limited number of previously published reports included patients after immunotherapy failure [36]. Thus, the issue of the efficacy of PD-1 inhibitors combined with BV after PD-1 inhibitors failure and before allo-HSCT remains extremely relevant.

\section{Materials and methods}

\section{Study design}

We retrospectively evaluated 21 patients with $\mathrm{r} / \mathrm{r} \mathrm{cHL}$ treated with combination of anti-PD1 antibody nivolumab $(3.0 \mathrm{mg} / \mathrm{kg}$ or $40 \mathrm{mg}$; IV, $60 \mathrm{~min}$ infusion) and brentuximab vedotin $(1.8 \mathrm{mg} / \mathrm{kg}$; IV, 30 min infusion) in 3 week cycles until disease progression, unacceptable toxicity, or planned allo-HSCT. Doses of nivolumab varied due to the ongoing clinical study of the nivolumab $40 \mathrm{mg}$ efficacy [38]. The initiation of this study is related to previous nivolumab pharmacokinetics studies demonstrated that median PD-1 receptor occupancy on peripheral blood $\mathrm{CD} 3+\mathrm{T}$ cells from patients with melanoma treated at a dose of $0.1-10.0 \mathrm{mg} / \mathrm{kg}$ was similar for every dose level over $0.3 \mathrm{mg} / \mathrm{kg}$, independent of nivolumab concentrations [40]. There also were not correlation between the dose, efficacy and safety in clinical trials of anti-PD-1 antibodies across a range of solid malignancies [41].
Inclusion criteria were age over 18 years, a histologically confirmed diagnosis of classic Hodgkin lymphoma, relapsed or refractory after at least 3 previous lines of therapy, previous treatment with nivolumab. Patients who had previous allo-HSCT were excluded from the study. This study was performed in accordance with the Declaration of Helsinki and approved by the institutional review board. All enrolled patients gave written informed consent. This study was approved by RM Gorbacheva Memorial Insitute institutional review board (IRB protocol No 24\13.01.2017).

The response was assessed by positron-emission tomography/computed tomography (PET/CT) using LYmphoma Response to Immunomodulatory therapy Criteria (LYRIC) and Lugano criteria. All patients that received at least one cycle of therapy were included in the efficacy and safety analysis. Safety was evaluated by registration of adverse events (AEs) according to Common Terminology Criteria for Adverse Events (CTCAE) v 4.03 criteria.

Primary chemoresistance was defined either by progression at any time during first-line therapy and up to 3 months after the end of treatment, and/or by persistence of a PET positive residual mass, using the quantitative 5-point scale Deauville score. Early relapse was defined as time to treatment failure more than 3 months but less than 12 months after the end of first-line therapy.

\section{Statistics}

In this study the overall response rate (ORR), progressionfree survival (PFS), overall survival (OS), event-free survival (EFS), duration of response (DOR) and frequency of grade 3 or higher treatment-related AEs were analyzed. Overall response rate was defined as the proportion of patients with complete response (CR) or partial response (PR) in measurable lesions by LYRIC criteria within a timeframe of 12 months. Progression-free survival was defined as the time from the first Nivo + BV cycle initiation to disease progression, relapse, or death; OS was defined as the time from the first Nivo + BV cycle initiation to death from any reason; EFS was defined as the time from the first dose of combination therapy to disease progression, relapse, death or initiation of other therapy; DOR was defined as a time from initial objective response to a documented disease progression or death. In each survival outcome, data was censored at the date of the last contact for patients who have not experienced the events of interest during their follow-up.

The survival was estimated using the Kaplan-Meier method with $95 \%$ CIs estimates. The descriptive statistics methods were applied when appropriate. The impact of clinical factors on response was tested with Chi-square and Mann-Whitney $U$ tests. Data analysis was performed using $\mathrm{R}$ version 4.0.2 (2020-06-22) software. 


\section{Results}

\section{Patient's characteristics}

Twenty-one patients with $\mathrm{r} / \mathrm{r}$ cHL were included in this study between January 2017 and February 2020 in RM Gorbacheva Research Institute, Pavlov University. Demographics and baseline disease characteristics for all included patients are listed in Table 1. The median age at Nivo + BV initiation was 29 (range 22-39) years, 67\% of patients had primary refractory disease, and $14 \%$ relapsed within 1 year of frontline therapy. The majority of patients received BEACOPP $(n=9,43 \%)$ or ABVD $(n=9,43 \%)$ regimens as firstline treatment for cHL. All patients received nivolumab monotherapy before the Nivo + BV combination, 10 (48\%) patients were treated with BV and $8(38 \%)$ patients with ASCT. The dose of Nivo during Nivo + BV therapy was $3 \mathrm{mg} / \mathrm{kg}$ in $17(81 \%)$ patients and $40 \mathrm{mg}$ in $4(19 \%)$ patients, the dose of BV was $1.8 \mathrm{mg} / \mathrm{kg}$ in $21(100 \%)$ patients. Other details of Nivo + BV therapy are presented in Table 1 .

Table 1 Patient's and therapy characteristics

\begin{tabular}{ll}
\hline Patient's characteristics & $N=21$ \\
\hline Age at Nivo + BV initiation, median (range) & $29(22-39)$ \\
Sex (\%) & \\
Male & $12(57)$ \\
Female & $9(43)$ \\
Histology of cHL (\%) & \\
Nodular sclerosis & $16(76)$ \\
Mixed cellularity & $2(9.5)$ \\
Lymphocyte rich & $1(5)$ \\
Lymphocyte depleted & $2(9.5)$ \\
Disease stage at Nivo + BV initiation (\%) & \\
IV & $21(100)$ \\
Prior lines of therapy before Nivo + BV initiation (range) & $7(3-13)$ \\
Prior radiotherapy (\%) & $13(62)$ \\
Prior ASCT (\%) & $8(38)$ \\
Prior BV monotherapy (\%) & $10(48)$ \\
Prior combination with Nivo and chemotherapy (\%) & $7(33)$ \\
Status of the disease at Nivo + BV initiation (\%) & \\
Progressive disease & $17(81)$ \\
Indeterminate response & $2(9.5)$ \\
Partial response & $2(9.5)$ \\
B-symptoms (\%) & \\
Yes & $2(10)$ \\
No & $19(90)$ \\
Dose of Nivo in combined therapy (\%) & $17(81)$ \\
3 mg/kg & $4(19)$ \\
40 mg & $3(1-12)$ \\
The median of Nivo + BV cycles, median (range) & \\
\hline
\end{tabular}

\section{Efficacy}

All patients were included in the efficacy analysis. Median follow-up was 19 (range 9-47) months. The overall response rate was 57\%. According to LYRIC criteria, CR as the best response was achieved by $24 \%$ of patients, PR by $33 \%$ of patients, stable disease (SD) by $14 \%$ of patients, indeterminate response (IR) by $19 \%$ of patients and progressive disease (PD) by $10 \%$ of patients. According to Lugano criteria, the rate of CR, PR and SD was identical, while $29 \%$ of patients demonstrated PD (Table 2).

At the data cut-off, the median OS was not reached, 24-month OS was $80 \%$ (95\% CI 50-93\%). Median PFS was 12 months (95\% CI 8.0-34.0 mo) with 24-month PFS of $31 \%$ (95\% CI 12-53\%) (Fig. 1). In 12 patients with objective response to treatment, median DOR was 9.0 months (95\% CI 3.0-NA mo) (Supplementary Material Fig. 1S). Only $1(5 \%)$ patient was alive and free of disease progression with no additional treatment after Nivo + BV therapy with the median EFS of 5.0 months (95\% CI 4.0-6.0 mo) (Supplementary Material Fig. 2S).

Table 2 Overall response rate according to LYRIC and LUGANO criteria

\begin{tabular}{lcc}
\hline Response & Criteria & \\
\cline { 2 - 3 } & Lyric & Lugano \\
\hline Complete response & $23.8 \%$ & $23.8 \%$ \\
Partial response & $33.3 \%$ & $33.3 \%$ \\
Stable disease & $14.3 \%$ & $14.3 \%$ \\
Progression of disease & $9.5 \%$ & $28.6 \%$ \\
Indeterminate response & $19.0 \%$ & \\
\hline
\end{tabular}

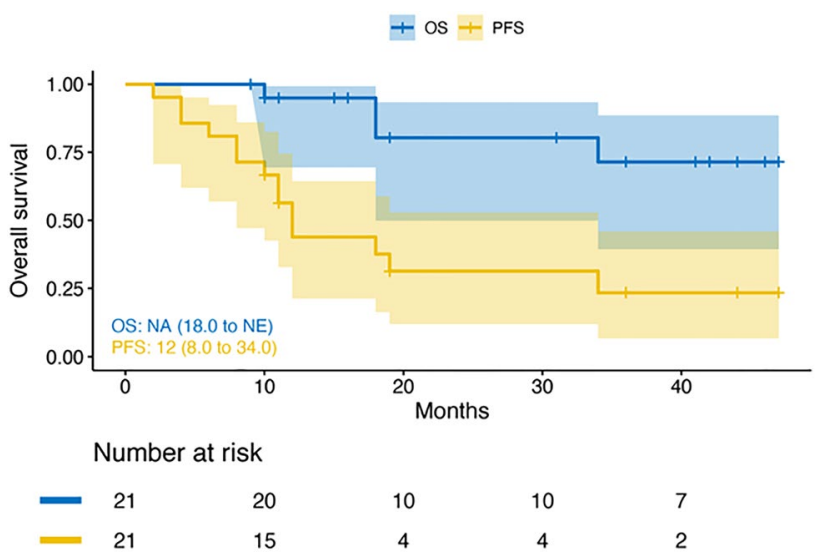

Fig. 1 Overall and progression-free survival after Nivo + BV therapy 


\section{Safety}

Adverse events of any grade were observed in 13 patients (62\%). Twelve patients (62\%) had grade 1-2 AEs and 2 patients (10\%) had 3-4 AEs. There were no cases of fatal toxicities. All cases of AEs are summarized in Table 3. The most common AEs were fatigue $(n=7,33 \%)$, elevated creatinine level $(n=7,33 \%)$, nausea $(n=5,24 \%)$, leukopenia $(n=4,19 \%)$ and infection $(n=4,19 \%)$. Grade $1-2$ infusion reactions were observed in $5 \%$ of patients. Grade $\geq 3$ AEs included 3 cases: grade 3 arthralgia and grade 3 pneumonia in the first patient, grade 4 thrombocytopenia, grade 3 anemia and leukopenia in the second patient. In 2 patients with grade $\geq 3$ AEs the Nivo + BV therapy was discontinued, treatment with $1 \mathrm{mg} / \mathrm{kg}$ methylprednisolone was initiated with complete resolution of observed AEs.

\section{Factors influencing the prognosis}

The influence of a set of clinical factors on PFS and ORR was analyzed (Supplementary Material Tables 1S, 2S). Taking into account that only 4 patients died, the statistical analysis of factors influencing OS was not possible. None of the assessed factors influenced patient survival or response rate. Time between initiation of Nivo + BV therapy and Nivo or BV monotherapy discontinuation did not affect the achievement of the response. The history of ASCT, BV and response to Nivo monotherapy also did not affect PFS. The trend towards statistical significance was found only for the number of therapy lines and response rates with the median number of $7.5(3.0-13.0)$ vs $5.0(3.0-9.0)$ in non-responders and responders respectively $(p=0.072)$.

\section{Follow up and additional treatment}

Nineteen patients (90\%) had additional therapy after Nivo + BV due to progression, relapse of the disease, or insufficient response (Fig. 2). Additional therapy was performed at the discretion of the treating physician based on the response to Nivo $+\mathrm{BV}$, number of prior therapies, availability of HLA matched donors, and the patient preference. Patients were treated with Nivo monotherapy in 5 (24\%) cases, BV monotherapy in $2(10 \%)$ cases, combination with Nivo and bendamustine in $8(38 \%)$ cases and Nivo + BV therapy in 1 case $(5 \%)$ of relapse after discontinuation of previous Nivo + BV therapy. Overall, 8 patients received allo-HSCT after Nivo + BV therapy at different time intervals. The median time before allo-HSCT was 8 months (range 5-21). The Nivo + BV combination as bridge therapy before allo-HSCT was conducted in $4(19 \%)$ patients. Donor was matched related in 2 patients, mismatched unrelated in 1 patient and haploidentical in 1 patient. Three out of four
Table 3 Adverse events during Nivo + BV therapy

\begin{tabular}{|c|c|c|c|c|c|c|}
\hline \multirow[t]{2}{*}{ Type of AE } & \multicolumn{2}{|c|}{ AEs overall } & \multicolumn{2}{|c|}{ AEs grade $1-2$} & \multicolumn{2}{|c|}{ AEs grade $3-4$} \\
\hline & $n$ & $\%$ & $n$ & $\%$ & $n$ & $\%$ \\
\hline Any & 12 & 57 & 12 & 57 & 2 & 10 \\
\hline Weakness & 7 & 33 & 7 & 33 & 0 & 0 \\
\hline Nausea & 5 & 24 & 5 & 24 & 0 & 0 \\
\hline Vomiting & 1 & 5 & 1 & 5 & 0 & 0 \\
\hline Diarrhea & 2 & 10 & 2 & 10 & 0 & 0 \\
\hline Headache & 2 & 10 & 2 & 10 & 0 & 0 \\
\hline Pruritus & 1 & 5 & 1 & 5 & 0 & 0 \\
\hline Rash & 1 & 5 & 1 & 5 & 0 & 0 \\
\hline Pyrexia & 3 & 14 & 3 & 14 & 0 & 0 \\
\hline Infections & 4 & 19 & 4 & 19 & 0 & 0 \\
\hline Peripheral polyneuropathy & 3 & 14 & 3 & 14 & 0 & 0 \\
\hline Palpitation & 1 & 5 & 1 & 5 & 0 & 0 \\
\hline Arthralgia & 2 & 10 & 1 & 5 & 1 & 5 \\
\hline Pneumonia & 1 & 5 & 0 & 0 & 1 & 5 \\
\hline Infusion reaction & 1 & 5 & 1 & 5 & 0 & 0 \\
\hline Dyspnea & 1 & 5 & 1 & 5 & 0 & 0 \\
\hline Anemia & 3 & 14 & 2 & 10 & 1 & 5 \\
\hline Leukopenia & 4 & 19 & 3 & 14 & 1 & 5 \\
\hline Thrombocytopenia & 2 & 10 & 1 & 5 & 1 & 5 \\
\hline ALT/AST increased & 3 & 14 & 3 & 14 & 0 & 0 \\
\hline Acute kidney injury & 7 & 33 & 7 & 33 & 0 & 0 \\
\hline Hypothyroidism & 3 & 14 & 3 & 14 & 0 & 0 \\
\hline
\end{tabular}




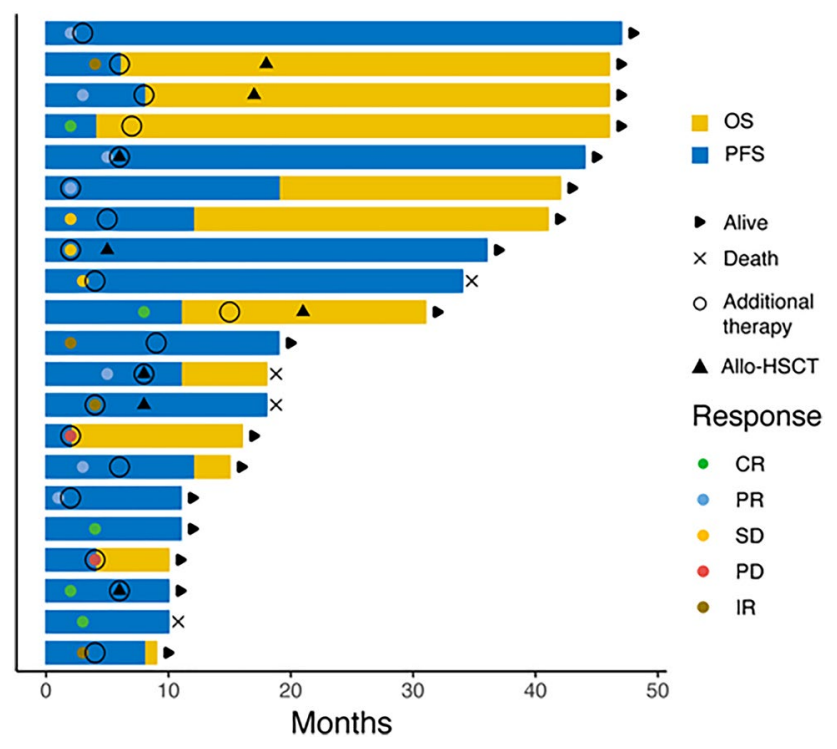

Fig. 2 Outcomes of patients after Nivo + BV therapy. Allo-HSCT allogeneic stem cell transplantation, $C R$ complete remission, $P R$ partial emission, $S D$ stable disease, $P D$ progressive, $I R$ indeterminate

patients remained in complete remission after allo-HSCT and 1 patient relapsed 4 months after allo-HSCT. Grade 4 acute graft-versus-host disease (aGVHD) (grade 4 gut, grade 3 liver and grade 3 skin) occurred in 1 patient after early withdrawal of immunosuppressive therapy due to decreasing donor chimerism. The patient responded to the combined immunosuppressive therapy (glucocorticoids, everolimus, ruxolitinib, etanercept). Chronic GVHD (cGVHD) occurred in 3 out of 4 patients including 2 cases of extensive cGVHD and 1 case of localized cGVHD. In case of relapse after allo-HSCT the therapy with brentuximab vedotin and donor lymphocyte infusion were used.

A total of $4(19 \%)$ patients died, including 2 after alloHSCT. The causes of death were secondary myelodysplastic syndrome $(n=1)$, COVID-19 infection $(n=1)$, severe poor graft function after allo-HSCT transplant hypofunction and infection $(n=1)$, and PD $(n=1)$.

\section{Discussion}

Treatment of patients with cHL after immunotherapy failure represents an unmet medical need, and defining the therapeutic strategy in this population is highly important. The aim of this study was to assess the potential of the combination of immune checkpoint inhibitors and BV as salvage treatment in severely pretreated population of patients with $\mathrm{r} / \mathrm{r} \mathrm{cHL}$. The combination of Nivo and BV has already demonstrated to be effective in patients with $\mathrm{r} / \mathrm{r} \mathrm{cHL}$ with ORR and CR of $82 \%$ and $61 \%$, respectively [32]. However, prior treatment with immunotherapy was the exclusion criteria in previously published studies. In the current study, all patients $(n=21)$ received Nivo monotherapy before the Nivo + BV combination and 10 patients were previously treated with BV. Moreover, 33\% of patients were earlier treated with other combinations of Nivo with chemotherapy before Nivo + BV. Our study demonstrates that the Nivo + BV therapy is a potentially effective approach in this heavily pretreated patients population with $57 \%$ overall and $24 \%$ complete response rate. While BV retreatment, consistent with published data, was beneficial in patients with $\mathrm{r} / \mathrm{r}$ cHL who had previously responded to BV therapy [42], only 3 out of 10 patients in current population had objective response to $\mathrm{BV}$ monotherapy previously. Considering that only 6 (29\%) patients responded to Nivo monotherapy the results of the study may demonstrate the possible synergism of immunotherapy and targeted therapy with BV. The lower response rates, as well as CR rate, in comparison with earlier study of this combination [32], are most likely associated with differences in patient groups (previous treatment with Nivo, BV, multiple lines of therapy). Despite the acceptable response rate, Nivo $+\mathrm{BV}$ therapy provides only a small chance of long-term remission. Allo-HSCT can be performed after response to Nivo + BV combination to consolidate the response and give a patient the possibility of cure [22].

The Nivo + BV combination demonstrated acceptable toxicity with development of grade 3-4 AEs in only $10 \%$ of patients. It is worth noting that grade 1-2 infusion reactions were reported only in 5\% of patients in comparison with previously published data on $44 \%$ of this AE incidence. The difference in non-severe AE can be explained by the retrospective collection of medical data in current study as opposed to prospective clinical trial safety assessments. All cases of AEs were controlled and adequately responded to conducted therapy.

Our study did not show a statistically significant effect of any clinical factors on the overall response rate and survival after Nivo + BV therapy including prior Nivo and BV monotherapy presence. There was only a tendency towards decreased response rates in more pretreated patients. This is most likely connected with a small number of study participants, as well as the presence of additional therapy after Nivo + BV almost in all patients (90\%).

In this study, the LYRIC criteria were used to allow avoiding early discontinuation of therapy in patients with tumor pseudoprogression when this therapy can be effective in the future [43]. Moreover, LYRIC criteria have been prospectively analyzed earlier in real clinical practice in patients with $\mathrm{r} / \mathrm{r} \mathrm{cHL}$ [21] and similar ORR and DOR were demonstrated with studies using Lugano criteria.

Limitations of this report include a retrospective study design within a single institution, which may lead to the selection bias, limited number of patients, lack of comparator 
arm and the significant proportion of patients that received additional treatment after Nivo + BV therapy.

\section{Conclusion}

In conclusion, the combination of nivolumab with brentuximab vedotin in $\mathrm{r} / \mathrm{r} \mathrm{cHL}$ after failure of nivolumab monotherapy is a potentially effective and safe approach and can be considered as bridge therapy before allo-HSCT in selected patients. Future studies with longer follow-up and expanded patient population are needed to determine the place of this combination in therapy of $\mathrm{r} / \mathrm{r} \mathrm{cHL}$.

Supplementary Information The online version contains supplementary material available at https://doi.org/10.1007/s10147-021-02085-6.

Author contributions Conception and design: FLV, LKV, MNB, MIS, and KAD. Provision of study materials or patients: FLV, LKV, MNB, $\mathrm{KAV}, \mathrm{BVV}, \mathrm{BAV}, \mathrm{BES}$, and PMO. Collection and assembly of data: FLV, LKV, MNB, and KPV. Data analysis and interpretation: FLV, LKV, MNB, VNP, KPV, and PMO. Manuscript writing: FLV, LKV, and VNP. Final approval of manuscript: all authors. Accountable for all aspects of the work: FLV and LKV.

Funding No additional funding.

Data availability Not applicable.

Code availability Not applicable.

\section{Declarations}

Conflict of interest All authors declare that they have no conflict of interest.

Ethical approval This study was performed in accordance with the 1964 Helsinki declaration and approved by the institutional review board. All enrolled patients gave written informed consent.

Consent to participate Not applicable.

Consent for publication Not applicable.

\section{References}

1. National Cancer Institute Surveillance Epidemiology and End Results Program (2019). Cancer stat facts: Hodgkin lymphoma. SEER https://seer.cancer.gov/statfacts/html/hodg.html

2. Kuruvilla J, Keating A, Crump M (2011) How I treat relapsed and refractory Hodgkin lymphoma. Blood 117(16):4208-4217. https://doi.org/10.1182/blood-2010-09-288373

3. Armitage JO (2010) Early-stage Hodgkin's lymphoma. N Engl J Med 363(7):653-662. https://doi.org/10.1056/NEJMra1003733

4. Kuruvilla J (2009) Standard therapy of advanced Hodgkin lymphoma. Hematol Am Soc Hematol Educ Program. https://doi.org/ 10.1182/asheducation-2009.1.497
5. Andre' M, Henry-Amar M, Pico JL et al (1999) Comparison of high-dose therapy and autologous stem-cell transplantation with conventional therapy for Hodgkin's disease induction failure: a case-control study. J Clin Oncol. https://doi.org/10.1200/JCO. 1999.17.1.222

6. Linch DC, Winfield D, Goldstone AH et al (1993) Dose intensification with autologous bone-marrow transplantation in relapsed and resistant Hodgkin's disease: results of a BNLI randomised trial. Lancet 341(8852):1051-1054. https://doi.org/10.1016/01406736(93)92411-1

7. Schmitz N, Pfistner B, Sextro M et al (2002) Aggressive conventional chemotherapy compared with high dose chemotherapy with autologous haemopoietic stem-cell transplantation for relapsed chemosensitive Hodgkin's disease: a randomised trial. Lancet 359(9323):2065-2071. https://doi.org/10.1016/S0140-6736(02) 08938-9

8. Josting A, Rudolph C, Mapara M et al (2005) Cologne high-dose sequential chemotherapy in relapsed and refractory Hodgkin lymphoma: results of a large multicenter study of the German Hodgkin Lymphoma Study Group (GHSG). Ann Oncol 16:116-123. https://doi.org/10.1093/annonc/mdi003

9. Weniger MA, Tiacci E, Schneider S et al (2018) Human CD30+ B cells represent a unique subset related to Hodgkin lymphoma cells. J Clin Investig 128:2996-3007. https://doi.org/10.1172/ JCI95993

10. Carey CD, Gusenleitner D, Lipschitz M et al (2017) Topological analysis reveals a PD-L1-associated microenvironmental niche for Reed-Sternberg cells in Hodgkin lymphoma. Blood 130:24202430. https://doi.org/10.1182/blood-2017-03-770719

11. Borchmann S, von Tresckow B (2017) Novel agents in classical Hodgkin lymphoma. Leuk Lymphoma 58(10):2275-2286. https:// doi.org/10.1080/10428194.2017.1300898

12. Glimelius I, Diepstra A (2017) Novel treatment concepts in Hodgkin lymphoma. J Intern Med 281:247-260. https://doi.org/10. 1111/joim. 12582

13. Gardai SJ, Epp A, Law C-L (2015) Brentuximab vedotin-mediated immunogenic cell death. Cancer Res. https://doi.org/10.1158/ 1538-7445.AM2015-2469

14. Cao A, Heiser R, Law CL et al (2016) Auristatin-based antibody drug conjugates activate multiple ER stress response pathways resulting in immunogenic cell death and amplified T-cell responses. Cancer Res. https://doi.org/10.1158/1538-7445. AM2016-4914

15. Younes A, Gopal AK, Smith SE et al (2012) Results of a pivotal phase II study of brentuximab vedotin for patients with relapsed or refractory Hodgkin's lymphoma. J Clin Oncol 30(18):2183-2189. https://doi.org/10.1200/JCO.2011.38.0410

16. Chen R, Gopal AK, Smith SE et al (2016) Five-year survival and durability results of brentuximab vedotin in patients with relapsed or refractory Hodgkin lymphoma. Blood 128(12):1562-1566. https://doi.org/10.1182/blood-2016-02-699850

17. Pardoll DM (2012) The blockade of immune checkpoints in cancer immunotherapy. Nat Rev Cancer 12(4):252-264. https://doi.org/ $10.1038 / \mathrm{nrc} 3239$

18. Armand P, Engert A, Younes A et al (2018) Nivolumab for relapsed/refractory classic Hodgkin lymphoma after failure of autologous hematopoietic cell transplantation: extended followup of the multicohort single-arm phase II CheckMate 205 trial. J Clin Oncol 36(14):1428-1439. https://doi.org/10.1200/JCO.2017. 76.0793

19. Chen R, Zinzani PL, Fanale MA et al (2017) Phase II study of the efficacy and safety of pembrolizumab for relapsed/refractory classic Hodgkin lymphoma. J Clin Oncol 35(19):2125-2132. https:// doi.org/10.1200/JCO.2016.72.1316

20. Lepik KV, Mikhailova NB, Moiseev IS et al (2019) Nivolumab for the treatment of relapsed and refractory classical Hodgkin 
lymphoma after ASCT and in ASCTnaïve patients. Leuk Lymphoma 60(9):2316-2319. https://doi.org/10.1080/10428194.2019. 1573368

21. Kozlov AV, Kazantzev IV, Iukhta TV et al (2019) Nivolumab in pediatric Hodgkin's lymphoma. Cell Ther Transplant 8(4):41-48. https://doi.org/10.18620/ctt-1866-8836-2019-8-4-41-48

22. Sureda A, Canals C, Arranz R et al (2012) Allogeneic stem cell transplantation after reduced intensity conditioning in patients with relapsed or refractory Hodgkin's lymphoma. Results of the HDR-ALLO study - a prospective clinical trial by the grupo español de linfomas/trasplante de médula osea. Haematologica 97(2):310-317. https://doi.org/10.3324/haematol.2011.045757

23. Ramos CA, Grover NS, Beaven AW et al (2020) Anti-CD30 CAR-T cell therapy in relapsed and refractory Hodgkin lymphoma. J Clin Oncol 38(32):3794-3804. https://doi.org/10.1200/ JCO.20.01342

24. Lepik KV, Mikhailova KV, Kondakova EV et al (2020) A study of safety and efficacy of nivolumab and bendamustine (NB) in patients with relapsed/refractory Hodgkin lymphoma after nivolumab monotherapy failure. Hemasphere. https://doi.org/10. 1097/HS9.0000000000000401

25. Rossi C, Gilhodes J, Maerevoet M et al (2018) Efficacy of chemotherapy or chemo-anti-PD-1 combination after failed anti-PD-1 therapy for relapsed and refractory Hodgkin lymphoma: a series from LYSA centers. Am J Hematol 93(8):1042-1049. https://doi. org/10.1002/ajh.25154

26. LaCasce AS, Bociek RG, Sawas A et al (2018) Brentuximab vedotin plus bendamustine: a highly active first salvage regimen for relapsed or refractory Hodgkin lymphoma. Blood 132(1):40-48. https://doi.org/10.1182/blood-2017-11-815183

27. Broccoli A, Argnani L, Botto B et al (2019) First salvage treatment with bendamustine and brentuximab vedotin in Hodgkin lymphoma: a phase 2 study of the Fondazione Italiana Linfomi. Blood Cancer J 9(12):100. https://doi.org/10.1038/s41408-019-0265-x

28. Lazzari C, Karachaliou N, Bulotta A et al (2018) Combination of immunotherapy with chemotherapy and radiotherapy in lung cancer: is this the beginning of the end for cancer? Ther Adv Med Oncol 10:1-12. https://doi.org/10.1177/1758835918762094

29. Peng J, Hamanishi J, Matsumura N et al (2015) Chemotherapy induces programmed cell death-ligand 1 overexpression via the nuclear factor-kB to foster an immunosuppressive tumor microenvironment in ovarian cancer. Cancer Res 75:5034-5045. https:// doi.org/10.1158/0008-5472.CAN-14-3098

30. Liu WM, Fowler DW, Smith P et al (2010) Pre-treatment with chemotherapy can enhance the antigenicity and immunogenicity of tumours by promoting adaptive immune responses. $\mathrm{Br} \mathrm{J}$ Cancer 102:115-123. https://doi.org/10.1038/sj.bjc.6605465

31. Kearl TJ, Jing W, Gershan JA et al (2013) Programmed death receptor-1/ programmed death receptor ligand-1 blockade after transient lympho-depletion to treat myeloma. J Immunol 190:5620-5628. https://doi.org/10.4049/jimmunol.1202005

32. Herrera AF, Moskowitz AJ, Bartlett NL et al (2018) Interim results of brentuximab vedotin in combination with nivolumab in patients with relapsed or refractory Hodgkin lymphoma. Blood 131(11):1183-1194. https://doi.org/10.1182/ blood-2017-10-811224

33. Advani RH, Moskowitz AJ, Bartlett NL et al (2018) Phase $1 / 2$ study of brentuximab vedotin in combination with nivolumab in patients with relapsed or refractory classic Hodgkin lymphoma: part 3 (concurrent dosing) results and updated progression-free survival results from parts 1 and 2 (staggered dosing). Blood 132(Suppl. 1):1635. https://doi.org/10.1182/ blood-2018-99-110002

34. Diefenbach CS, Hong F, David K et al (2017) Safety and efficacy of combination of brentuximab vedotin and nivolumab in relapsed/refractory Hodgkin lymphoma: a trial of the ECOGACRIN cancer research group (E4412). Hematol Oncol 35:84-85. https://doi.org/10.1002/hon.2437_72

35. Diefenbach CS, Hong F, David KA et al (2016) Title: a phase I study with an expansion cohort of the combination of ipilimumab and nivolumab and brentuximab vedotin in patients with relapsed/ refractory Hodgkin lymphoma: a trial of the ECOG-ACRIN cancer research group (E4412 Arms D and E). Blood 128:1106. https://doi.org/10.1182/blood.V128.22.1106.1106

36. Diefenbach CS, Hong F, Ambinder R et al (2019) Extended follow-up of a phase I trial of ipilimumab, nivolumab and brentuximab vedotin in relapsed Hodgkin lymphoma: a trial of the ECOG-ACRIN research group (E4412). Hematol Oncol 37:123124. https://doi.org/10.1002/hon.83_2629

37. Diefenbach CS, Hong F, Cohen JB et al (2015) Preliminary safety and efficacy of the combination of brentuximab vedotin and ipilimumab in relapsed/refractory Hodgkin lymphoma: a trial of the ECOG-ACRIN cancer research group (E4412). Blood 126:585. https://doi.org/10.1182/blood.V126.23.585.585

38. Lepik KV, Fedorova LV, Kondakova EV et al (2020) A phase 2 study of nivolumab using a fixed dose of 40mg (Nivo40) in patients with relapsed/ refractory Hodgkin lymphoma. Hemasphere. https://doi.org/10.1097/HS9.0000000000000480

39. Diefenbach CS, Hong F, Ambinder RF et al (2020) Ipilimumab, nivolumab, and brentuximab vedotin combination therapies in patients with relapsed or refractory Hodgkin lymphoma: phase 1 results of an open-label, multicentre, phase 1/2 trial. Lancet Haematol 7(9):e660-e670. https://doi.org/10.1016/S2352-3026(20) 30221-0

40. Agrawal S, Feng Y, Roy A et al (2016) Nivolumab dose selection: challenges, opportunities, and lessons learned for cancer immunotherapy. J Immunother Cancer. https://doi.org/10.1186/ s40425-016-0177-2

41. Wang X, Feng Y, Bajaj G et al (2017) Quantitative characterization of the exposure-response relationship for cancer immunotherapy: a case study of nivolumab in patients with advanced melanoma. CPT Pharmacometr Syst Pharmacol 6:40-48

42. Fukuharaa N, Yamamotob G, Tsujimurac H et al (2019) Retreatment with brentuximab vedotin in patients with relapsed/refractory classical Hodgkin lymphoma or systemic anaplastic large-cell lymphoma: a multicenter retrospective study. Leuk Lymphoma. https://doi.org/10.1080/10428194.2019.1654100

43. Cheson BD, Ansell S, Schwartz L et al (2016) Refinement of the Lugano classification lymphoma response criteria in the era of immunomodulatory therapy. Blood 128(21):2489-2496. https:// doi.org/10.1182/blood-2016-05-718528

Publisher's Note Springer Nature remains neutral with regard to jurisdictional claims in published maps and institutional affiliations. 